\title{
The predicate fabric of abstraction: the hard test of logical inversion
}

\author{
Konstantinos G. Papageorgiou \\ Ionian University, Department of Informatics, \\ University of Athens, Applied Philosophy Lab \\ cconstantinoss@gmail.com \\ Demetrios E. Lekkas \\ Hellenic Open University, \\ Patras Greece \\ ja-dim@hotmail.com
}

\section{Abstract}

he paper starts with an ultra-compact deposition on the two ubiquitous complementary dual pairwise organized methodological procedures of episteme, i.e. the analytic method (analysis - synthesis) $\&$ the abstract process (abstraction - structure). Next, the authors examine some ground rules and concepts pervading causality and inference and their junctions, attempting to discriminate between information flow in empiricism and theoretical causality of proof; only then is a connection between them attempted and investigated. In the authors' effort to establish a consistent theoretical outlook, if not approach, the technique of logical inversions is also used as a partial yet powerful guide elucidating how successful their attempts were. Apart from clarifying some opaque concepts in logic, in set theory and in the staple empiricism of science, this paper also sets the stage for questioning whether some grave flaws could be located in traditional, save ill-founded, notions in hardcore science, on occasion of the par excellence typical example of fundamental and never challenged approaches in physics. The fact that something has been accepted as holding does not at all mean that cracks may not be located in its epistemological makeup at some posterior time. And it is the text's task here to ask some painful questions and try to set some realistic boundaries to things by aptly utilizing available irresistible standard «tricks» from logic and from the classical scientific method and from reverting to fruitful techniques and to telling examples, pushing hard for convincing answers. 
Keywords: Science, empiricism, logical inference, logical inversion, analysis - synthesis, abstraction - structure, cause and effect

\section{$\Pi \varepsilon \rho i \lambda \eta \psi \eta$}

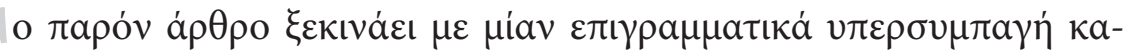

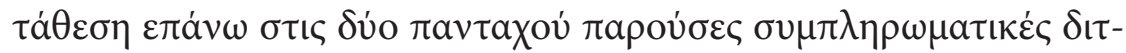

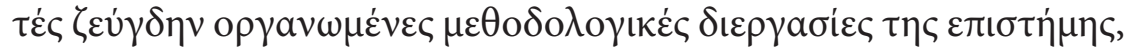

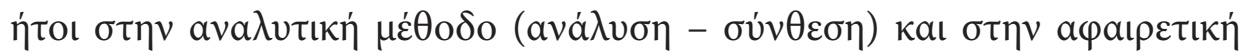

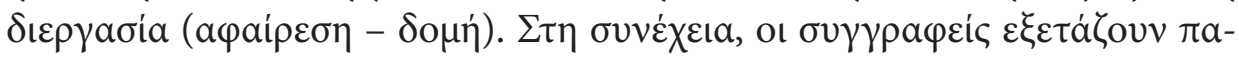

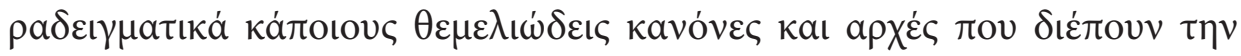

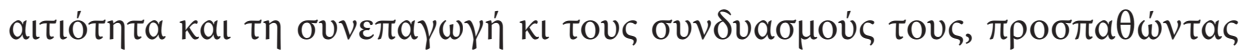

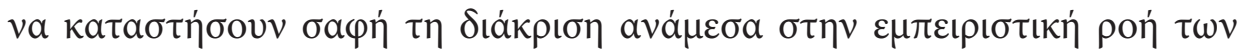

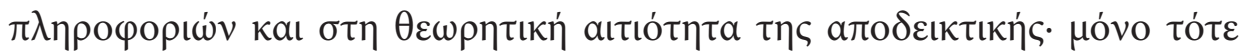

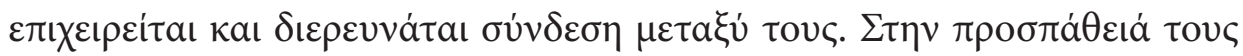

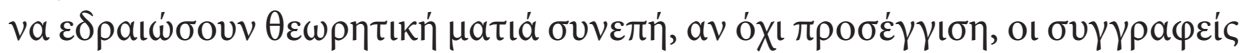

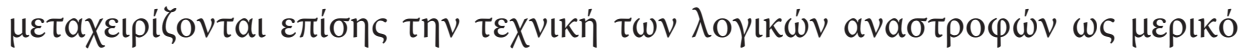

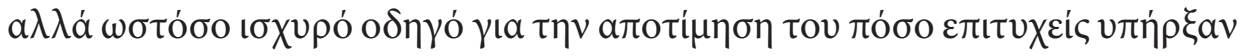
o a a

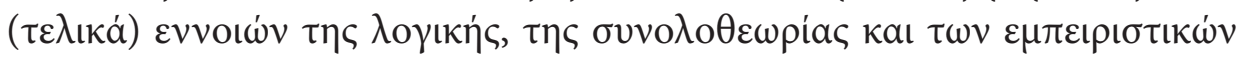

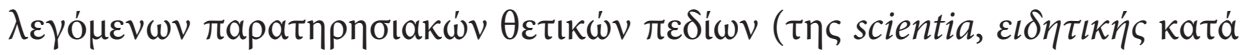

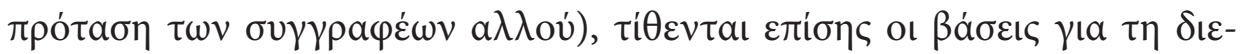

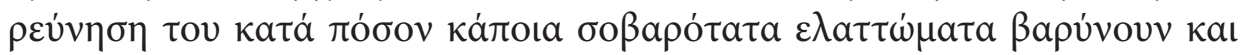

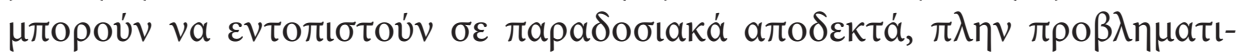

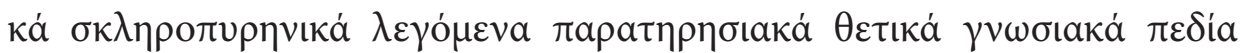

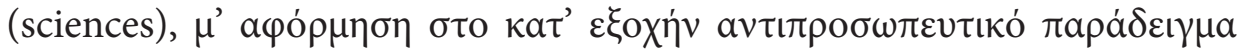

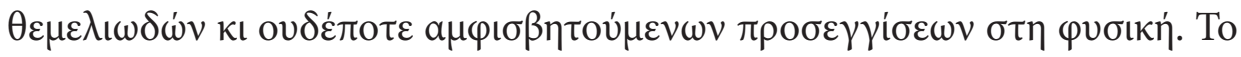

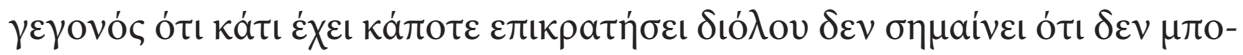

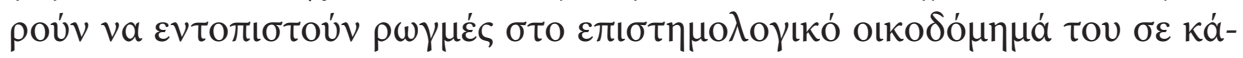

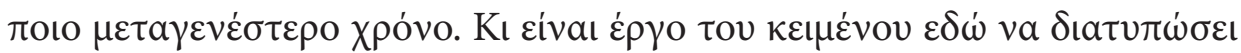

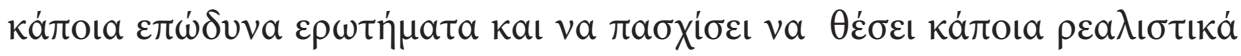

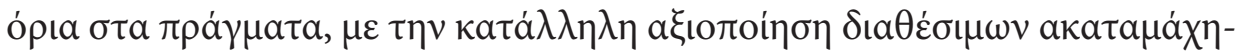

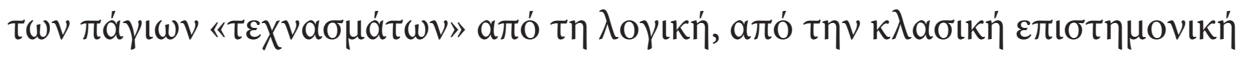

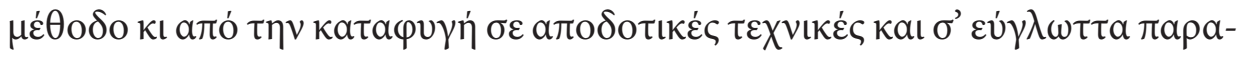

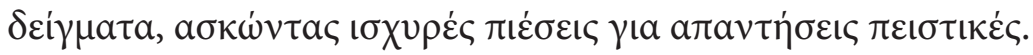




\section{Introduction}

The purpose of this paper is twofold.

On one hand, its first purpose is to demonstrate the priority of logic - an almost forgotten principle of both applied and theoretical sciences. Science is an imperialist, exclusive, authoritarian, powerful, controlling system (Collins \& Evans, 2002; Latour \& Woolgar, 1986), as expressed by "expert scientists" who have every reason to endorse data-driven statistics, but (eventually) do not have enough reason to focus on an absolute priority of abstract argument-driven logic and not violate it. This type of attitude, i.e. the one pervading leading contemporary science, relishes in mixing up and haphazardly clouding up logical orderliness of arguments with empirical observation of objects and phenomena, i.e. priorities between methodology and description. In so doing, it has landed up on a clever way of excluding "non-experts" from expressing any opinion about each science's assumptions -let alone from doubting, challenging or proposing reforms regarding statutory matters. But this is against episteme's basic / statutory values. Thus, the required validity of the form of building arguments gets mixed up with their content; this constitutes a treacherous state of affairs which has been known, pointed out and studied in logic, starting from the so-called philosophical logic and rhetoric since classical Greek and Roman antiquity. The difference between science and episteme has been discussed elsewhere. With logic alone, in the proper sense of the concept, one may delve very deeply into the sciences and even propose revolutionary reforms.

On the other hand, the second purpose of this paper and of the ones following is to underline the necessity and perhaps delineate a core direction towards proposing statutory changes in logic as a system itself. Formal / symbolic logic evolved from the 19th century on with the ability to serve applications, which are rigorously prescribed and described within the formulation; developing any theoretical system not in pure abstraction but in association to practical applications, or, worse, with practical applications as a main focal purpose is inherently problematic, i.e. ideologically asymmetrical, partial and even sketchy at times. 


\section{Analyses and abstractions}

As was discussed in Papageorgiou \& Lekkas (2018), following what has first been laid out in an exemplary way in Lekkas (2003), the theoretical method of science and its entire context (or the epistemonic method) involves two complementary self-coupling procedural tools; the analytic method and the abstractive process (Lekkas, 2003; Papageorgiou \& Lekkas,

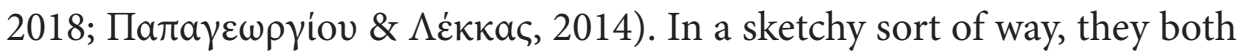
involve a mutual auto-reverse pair of constituent procedures each, ideally obeying some basic guidelines.

- The analytic method involves two stages of mutually opposite senses, analysis and synthesis, subject to the following three rules of thumb: no analysis without synthesis, no synthesis without analysis, no starting synthesis before completion of analysis. In this sense: analysis is a breakup into constituents, whereas synthesis is a (re)composition back towards the integral object or procedure or phenomenon and effectively constitutes a return from an accomplished and complete analysis.

- The abstractive process involves two stages of mutually opposite senses, abstraction and structure, subject to the following three rules of thumb: no abstraction without structure, no structure without abstraction, no starting structure before completion of abstraction. In this parallel sense: abstraction is a gradual inclusion of an element in ever-wider supersets starting from inclusion in the singleton or unit set, along with the classifying criterion of including something into a set, rising in ever-wider including sets (and types and categories for those more favourable to the corresponding particular theoretical setups), reflecting properties and/ or qualities, following upon a stage-by-stage path of generalization leading to some terminal level of total / absolute abstraction, whereas synthesis is a route of (re)specialization back towards the concrete singleton including one elementary object or procedure or phenomenon (and thence potentially to its content, which is now a single "element" or "member" in set-theoretical language), and effectively constitutes a return from an accomplished and complete abstraction.

These two pairwise organized methodological procedures actually face in mutually opposite directions, having a shared dual conjugate starting 
point, contingent on the oftentimes hard and treacherous dialectics of the duality between element and singleton and of judgment or establishment or agreement or convention regarding its status. However, from then on, they deviate from each other in anti-diametric senses, as was already said, a. analysis running towards a stage by stage segmentation and b. abstraction running towards a step by step inclusion in logic-theoretical wider inclusive supersets (a concept also pretty much expressed in the relevant terminology as predicates).

For example, if the starting point is a house, in analysis one would e.g. break it up into materials such as bricks, concrete, wood etc., or sections such as floors, rooms, kitchens, staircases, or constituent parts such as windows, doors and walls etc., whereas, in generalizing abstraction, one would include it in categories, e.g. urban complexes, or degrees of architectural artistic value and elegance, or placement in considerations of tradition and modernity, or agreement with respect to its fulfilling criteria of comfort, of class etc. etc. On the other hand, conversely so to speak, synthesis would be the process employed for going from e.g. materials or sections or constituent parts (windows, doors and walls) to entire houses, whereas structure would be the corresponding specializing process of going from e.g. the properties or sets or categories, actually serving here as predicates (e.g. of modern and urban), back to a single particular house in question.

\section{Of eggs and hatchlings}

Here, though, we come across a crucial elementary discussion of tantamount importance in the relationship and tradeoffs between some hardcore workings of logic in organized rational formal methodical thinking. These observations come with full ramifications and repercussions on the ground issues pervading theory and empiricism, mathematics and the ("exact") sciences, cause and effect, necessity and sufficiency, causality and potentiality as in (required / necessary? ample / sufficient? possible / potential?) cause and effect, discussion and data and conclusions, fact and information and fantasy, directionality of the arrows of inference, evidence and proof, all subjects slippery and sticky.

Let us start from a very old very familiar example about the hatchling baby bird in general, or, say, of "the chicken" in particular. As we all know, 
by natural law pervading all our experience with living things, no chicken comes to this life unless it has grown as a fetus in an egg. Therefore, the hatched egg is a necessary condition for the chicken to be born and it is safe to conclude, once we see a chicken, that there was an egg in which the chicken once was and that, without that egg, our baby could not have existed. It is understood, of course, that the condition is not sufficient; there are eggs that do not lead to chickens because they are not fertilized, or they have not been hatched, or they have been cracked, or the hatching has not been successful for whatever reason. Therefore, the egg is a potentiality for the chicken.

So, this is the biological aspect of things: egg is a necessary but not sufficient cause for a hatchling chicken, a sine qua non as a depot of mechanism, but only potential as termination or purpose of the said mechanism, and the chicken is a potential effect. However, once one sees the chicken, one has ample information to make a secure prediction for an egg of provenance. What are we saying? That, on the level of information as regards source, seeing the chicken (the effect of biological birth) is a secure cause for information. And that is a prima facie reversal of apparent causality pervading the empiricism of observational sciences. How do I know? Simple: I see the effect of the mechanism which is the cause of my information. Bingo! All is ready now for me to get theoretical and empirical causality reversed.

\section{Triggering logical inversions}

First of all, it should be pointed out that abstract logic, with its concluding and proving (and disproving) methods, is a test in tautology, or expressive identity of something stated, regardless of "meaning": in deeper essence, a proof is a check of logical and/or factual equivalence, namely of essentially saying the same thing in two alternative sets of propositions, regardless of the semantics -or "meaning" of the statement-. In an axiomatic theory, all the theorems are tautologous to the full set of the axioms and their combinations and the associated definitions. Also, let us bear in mind that, following singular statements, an infinity of levels of generalization and specialization follow in a series starting with Predicate Logic (of "level 1"), setting forth a model of single-stage generalization -via catego- 
ry-marker universal quantifier $\forall$, for all $x$ - and its inverse one-stage specialization -via object- or process-focusing marker existential quantifier $\exists$, there exists (at least) one $x$ such that-.

Logical inversion by a simple wide-spread scheme known in ancient Latin "philosophical logic" as modus tollens is a good valid way for checking up on the generalizing / specializing process; following upon a tautologous expressive application of the axioms and definitions as it should, it lays out mentally stimulating checks regarding the tautology of converting all affirmations to negations and vice versa while, simultaneously, turning the syntactic order of the sentence around. In fact, logical inversions by modus tollens are but an exemplary application field of simple predicate first order- logic. They are as good as predicate logic is; they are bound to predicate logic and possible weaknesses in predicate logic reflect fully on the possibility to apply such inversions in the first place.

Logically inverted sentences are tautologies and, oftentimes, the problem of the initial sentence is better demonstrated in its logical inversion. For example:

- "Everyone that is not with us is against us" is tautologous to

- "Everyone that is not against us is with us"

Here, the nonsense of the statement is established by making us realize that its combination with a conjugate statement meaning precisely the same thing leads to logical indeterminacy due to inconsistent semantic overlap. Simple as it may be, the process of modus tollens logical inversions is not only used to couple the universal and the existential quantifiers, but also has far-reaching implications when used as a (hard) test for categorical inclusion, as was already implied. The relation between the two quantifiers is expressed by the following logical inversion, incumbent in the fact that the former is established by inclusion of the universal quantifier $\forall$ in the axioms, whereas the latter is a statement of the very definition of the existential quantifier $\exists$ by means of a consistent combination of two axiomatic entities: the said universal quantifier and the negation operator $\neg$ :

i. by definition:

ii. by a theorem, validated by an elementary proof:

$$
\exists x=\neg \forall x \neg
$$$$
\forall x=\neg \exists x \neg
$$ 
And why is it that validity of inference, used in such predicate-bound cases of generalization and specialization and category, can be utilized in any inference, which may be applicable to one single example or case? The answer to this one is simple. Given that an inference within a proof is a check and establishment of a tautology, it regards ALL individual statements compliant to this formulation; our case at hand, then, is an ideal indistinct choice representative of anything compliant to the particular schematic typology, and that does already presuppose and incorporate an accomplished degree of generalization / specialization, which is the nitty-gritty of abstraction, which is what mathematics does and is in the first place. In simpler language, an implied generalization says that if A implies $\mathrm{B}$, or, in symbols, if $\mathrm{A} \Rightarrow \mathrm{B}$, it is understood that "every time A then B"; and if the implication (or inference or whatever name we choose for it) is necessary then there is a necessity implied in the generalization of predicate - let alone the fact that the Latin word prodicatum is but an acknowledged established Latin rendition of Greek syntactical term $\kappa \alpha \tau \eta \gamma o ́ \rho \eta \mu \alpha$ (catēgorē$\mathrm{ma}$ ); and, to whoever understands, that is that.

In what follows we shall examine some implications of employing logical inversions in the sciences. Some results, far-reaching and tremendously groundbreaking as they may be, only result from simple logical steps and they do so in an almost automatic fashion. Our examples mean only to stimulate and unboggle the mind, in an intent which is most suggestive, typical and representative, by no means methodical or exhaustive; we try to alert, not to sum up.

\section{CASE STUDY: the maggot-infested meat}

Every so often an old woman may notice that, as meat grows stale, it rots and/or mildew and maggots develop automatically. Who put them there? "They come out of nothing and nowhere, the meat itself creates them"; that is what some old people used to think in a magical way of looking at things. This may sound odd to us now, but only because we have been accustomed to the idea of micro-organisms and to the basic biological assumptions about reproduction -one being that spontaneous generation is impossible, especially a generation of evolved organisms of creatures singularly identifiable in the biological classification of the animal kingdom. Of course, 
Francisco Redi, back in the 17 th century, did demonstrate that maggots did not appear in meat kept in tightly closed containers.

Anyway, we positively know now that rotting and mildew are due to fungi and seeds are needed for them to grow and that eggs are a sine qua non prerequisite for a subsequent hatching of maggots. If they do appear (in nature), then, no doubt, some mother insect managed to visit the meat and lay miniature fertilized eggs perhaps not readily visible to the naked or untrained eye. It is time now to explore and develop the logic of the proposition:

"If there are maggots, there (certainly) have been eggs".

First observation possible here, but one that people do not always make, regards the temporal succession of events / observation implicit in the statement; it is notable that this succession is formally underrated and typically lost, if the symbolic formal representation of our observation is simply noted on paper or on the screen as a non-temporal explicit expression "'. We claim this is a treacherous simplicity that becomes a misleading, outright unscientific and unepistemonic obscurantism, and points in a direction of instant loss of focus, despite professing to be stating a simple self-evident intuitive and scientific fact. In this paper, we shall see more aspects of the relevance of the before - after temporal succession and its relevance in a systemic mechanism-bound attestation of the cause-and-effect succession. Let us mark this last word in particular; the sciences cannot be warned enough of the trap of back-staging or ignoring this principle.

Thus, before we go and unshroud the directionality of this series of remarks, let us lay out the unspecified formal development of this statement of ours and of its inversion which, only too often, can be caught just blabbering nonsense; and this is nonsense of which no one makes note, to which no one pays attention, which possibly no one realizes even:

Indeed, which side is cause and which side is effect here? What is happening, what do we understand, why are we stating what we are stating and how do we know, and says who? Is it a lucid fact that there have been fertilized eggs and that that is the only way for maggots to be here now? Could I have had eggs without maggots, and then how would I have found out? How could I possibly have known? Did I find eggs? Or am I securely establishing upon hard evidence, by the (now) common ("scientific") knowledge that an insect has laid eggs and that is why we see maggots now? Let us take our pick, by attempting to locate the "plausible" "causal" scheme, on both 
mutually contradictory sides of respective standard scientific addictions (empiricism and proof), which is legitimately inverted by the theorems.

eggs $\Rightarrow$ maggots (natural biological law) $\quad \Leftrightarrow$ no maggots $\Rightarrow$ no eggs???

maggots $\Rightarrow$ eggs ( $\mathrm{ABC}$ 's of empiricist science) $\Leftrightarrow$ no eggs $\Rightarrow$ no maggots???

Which is it now? Which is cause and which is effect here? And which is plain mechanical scientific fact and which is information theory? And which is necessary and which is sufficient? And why? And how can this mess be fixed? And why does no one talk about it? Is it because they spontaneously "make sense" on face value, despite the difference in context? And is context the institutional guarantor of spotting, stating and describing reality? Is it all of them, despite the fact that they are intuitively incongruous and logically mutually inconsistent? At the end of the day, does "making sense" make sense even?

In relevance, on another tangent course of thought, if both make sense and both hold, I ought to be able to complement the previous arrows with their inverses, to turn inferences into logical equivalences and modify the preceding scheme by writing:

eggs $\Rightarrow$ maggots (natural biological law) $\quad \Leftrightarrow$ no maggots $\Rightarrow$ no eggs

$\Leftrightarrow$
maggots $\Rightarrow$ eggs (ABC's of empiricist science) $\quad \Leftrightarrow$ no eggs $\Rightarrow$ no maggots

whence, in some cases that "make sense" (perhaps throughout the sciences):

$$
\begin{aligned}
{[\text { cause } \Leftrightarrow \text { effect }] \Leftrightarrow[\text { effect } \Leftrightarrow \text { cause }] } & \Leftrightarrow[\text { no cause } \Leftrightarrow \text { no effect }] \Leftrightarrow \\
& \Leftrightarrow[\text { no effect } \Leftrightarrow \text { no cause }]
\end{aligned}
$$

What difference is there in principle between this and the "whoever is not with me" bit? In what sense does this not lead to a generalized institutional sensible ambiguity of everything? And why is this new school of thought a theory of everything that is relative and uncertain (mark the wording as intentional please)? And does this not lead directly into a dark era of expert opinion reintroducing a new-age sorcery or a magical or eschatological or para-theological verbosity? Could we not do something as simple as demanding: 
- separate causalities for necessary and for sufficient?

- separate causalities for abstract / concrete and analytic / synthetic with STATED boundary each time?

- separate causalities for affirmative and negative, with a good law conjoining, reconciling and connecting the two via a good rigorous supra-structure?

- separate (mutually inverse) causalities for (theoretical proof-orientated / institutional conceptual systemic) episteme and/or for (practical verification-orientated / informational perceptual observational) science and for their validations and rejections?

- theoretical anatomy of causality once mutual independence of cause and effect has been secured, i.e. for making effectively and absolutely procedurally sure that a said "cause" is ruled out from being an (alleged?) effect of the effect, even partially, and that a said "effect" is ruled out from being an (alleged?) cause of the cause?

\section{Causality and arrows of inference}

Indeed, the question spontaneously arises: what is causal and what is casual here and in "exact" science and how? After aeons of darkness, both episteme and science came to shed light on the relationship between us, our organisms and the way we perceive things in our share of the biosphere. By definition, as regards all living creatures on this planet, we are all captive to our ways and abilities of perception and conception, or else we die; fittest means best adjusted. The alleged and attested breach between our senses, our sensory information processing, our pattern recognition, our conclusions, our reasoning, our modeling, our testing, our experimentation and our logic, our intellect, all this has its roots in intake, internalization and internal processing. We are living creatures, aren't we? Well, then, let's face it, that's what we are designed to do.

Some epistemological "schools of thought" think they are advanced and cool by dismissing the naïveté and backwardness of matching the incoming stimuli and information to the intrinsic processing, interpretation, testing and learning, through an interface of matching signal received to intuition to recognition. If any system of science or logic barges in triumphantly posing and drawing value and utility out of being "non-intuitive (and thence 
even counter-intuitive)", it is probably suspect of darkness. One should beware and perhaps fight against epistemonic and/or scientific schools of thought spitting out endless and uninhibited volumes of self-referential and self-indulgent and effete narratives and circular schemes, often cryptic ones, where ultimately A proves B because B proves A and so forth, all smothered in a murky sauce of people in closed circles who are experts at themselves and at each other. What makes no sense is neither epistemonic nor scientific nor true, in some applicable, proper and true sense of "true sense".

And, if logic gets a free pass at being not necessarily intuitive, that does not mean that is should be conceivable as necessarily not intuitive; and if something could, as a last resort, be non-intuitive, how can this statement be construed as freedom for a proposition to be counterintuitive? Says who? Says someone with a bipolar disorder that cannot tell among necessary and sufficient and unnecessary and insufficient and between rights and duties? Should such individuals not visit a specialist and solicit help?

In the good old humanistic ways of the world, logic shall be reason and reason shall be logic and it must be checked for proper behaviour and, wherever it gets caught ill-behaving, it shall be revised and rectified. Has it not been sufficiently redone already? Unfortunately, the authors do not think so at all and are all too aware of the gravity of this statement that they are making.

\section{Multipolar anatomy of logical inversions}

In this next technical section of this paper, then, the authors will lay out the framework and context and let the recipients of the text to think it out by themselves, without forcing any ready-made conclusions, but use this tribune as an impulse to think out. The maggot - egg statements and logical inversions to be checked, starting upon the occasion of this proposition, would form some minimal logical quadripoles to scrutinize, calling for the dialectics implicating some of their formal and contextual inversions.

Before checking necessities and inversions and alleged and/or real equivalences and tautologies, regardless of meanings and truths and sense-making and time sequences and precedences and causality arrows, we must make sure i. that we have two full quadripoles of four (4) statements each, ii. that the temporality and "certainty" (in fact conviction) of the statements wishes 
and tries hard to follow a bona fide route of "pre-established knowledge of institutional asymmetries", in that spotting of maggots leads to the conclusion that there certainly have been eggs in some past time, whereas detection of eggs leads to the conclusion that there may come to be maggots in some future time (make particular note of that) and, last but not least, the conjugate pair that ii.a that the first group A. of four start from observation, i.e. they are orientated in the proper old-fashion sense (both directional sense and reasonable sense made) of empiricism and of "exact" science (observation to experimentation to hypothesis to theory to law), whereas ii.b that the second group B. of four more start from sensible established legitimate scientific knowledge, i.e. they are orientated in the proper old-fashion sense (both directional sense and reasonable sense made) of bona fide established scientific knowledge of systemic mechanism starting with empiricism and application of law. If, now, someone already sees the philosophical and epistemological problem here, we sympathize; and if someone thinks it is all naive and resolved, they are welcome to their convictions.

The reader is requested to remark that what is in parentheses regarding timing and necessity / sufficiency are not parts of the formal statement, nor do they seem to be required except on an interpretation / application / meaning level, which changes everything in the scientific declaration and appraisal but does not seem to be required in the logical form of the statement. That happens simply because there are no propositional calculus modifiers of those sorts used in the "exact" sciences. The scientists practising them do however believe that they do indeed direct their arguments in this "logical" fashion and try to check their assertions by putting them on various theoretical "logical contraptions / machines" (cf. the Turing machine, especially in association to its equivalence to one of Goedel's historic theorems), letting it all boil down to whether the machine will eventually stop or be caught in an endless or never-terminating circle. And that is supposed to lead to adequate conclusions regarding truths or realities or some haphazard inadequately discriminated concoction of the two.

When does the "machine" establish untruth? Is it if it stops on a negation or refutation of some statement, or is it if it shall never stop? Meaning what? Do they amount to the same or not, and if they differ what is the nature of their difference? Does non-stopping establish a cul-de-sac or an undecidability? What is the correct establishment of degrees of certainty 
in the all-purpose, unconditional, time-orientated causal scientific syllogism -which is what scientific syllogisms try to be and are happy if they achieve this goal? And what is the current holding flow of time in a universe that purports itself to be what it is "thermodynamically"? And is that inescapable? And what happens if it isn't? And "where"? And what is the theoretical and factual difference between the pair "inputs / considerations - outputs / conclusions" and "cause - effect"? And is an untruth the same as a falsehood? Why or why not? Says who?

No matter who says, anyone will attest that we are made to recognize that a systemic mechanism will produce cause-and-effect in a connected associated conjugate repetitive before-after scheme, where cause is mobilized before and effect results after, and if, empirically speaking, I see something and identify it as an effect looking for its cause, I see it first and, upon completing investigation of my information-receiving source, I conclude thereupon, i.e. afterwards, something about what preceded (before), if I make the assessment that my depot and earliest possible source of stimulus is an effect of some cause that has preceded in the flow of things. We only react to stimuli thus because we are animals and we can't help it, that's how neuro-processors are designed, their speed being only finite and their function producing an observable effect; of what cause? The problem is obvious.

\section{The quadripoles of the maggot}

A.

1. If (there are?) maggots, then (there are? / there can be? / there must be? / there can have been? there must have been?) eggs.

2. If (there are?) maggots, then (there are? / there can be? / there must be? / there can have been? there must have been?) no eggs.

3. If (there are?) no maggots, then (still) (there are? / there can be? / there must be? / there can have been? there must have been?) eggs.

4. If (there are?) no maggots, then (there are? / there can be? / there must be? / there can have been? there must have been?) no eggs.

Here is the example of a direct application of predicate tautologous inversion by the axioms, definitions and theorems stated above. In particular, what is important is to show that in propositional logic any valid case has a valid inversion in the following tautology: 
- "Every time there are maggots, there are eggs" is tautologous with its logical inversion:

- "Every time there are no eggs, there are no maggots".

Thus, in the four numbered statements above, there is no tautology between any two by virtue of inversion, because the four statements present an independent quadripole of four independent statements. Let us now try to check the four systemic scientific and/or logical assertions reflecting possible laws by juxtaposing another four (4) independent logical clauses, constituting - as it is supposed to turn out- logical inversions of the preceding quadripole by virtue of modus tollens and therefore tautologous to it on a one-to-one basis.

$B$.

1. If (there are? / there have been?) eggs, then (there are? / there may be? / there can be? / there must be? / there can come to be? / there must come to be?) maggots.

2. If (there are? / there have been?) eggs, then (there are? / there may be? / there can be? / there must be? / there can come to be? / there must come to be?) no maggots.

3. If (there are? / there have been?) no eggs, then (still) (there are? / there may be? / there can be? / there must be? / there can come to be? / there must come to be?) maggots.

4. If (there are? / there have been?) no eggs, then (there are? / there can be? / there must be? / there can come to be? / there must come to be?) no maggots.

By logical inversions, in this loose sense, the "correct" correspondence would be:

\section{A.1. to B.4., A.2. to B.2., A.3. to B.3., A.4. to B.1.}

Are these, then, normal contents of the legitimate body of purported "logically rigorous" "scientific" "proofs"? And are proofs tautologies as they ought to be? Where? We shall let the reader check for themselves when, where, under what circumstances, in which formulation these pairs of statements say or do not say the same thing, and, if they don't, what is the difference, and why anyone should seriously take such proofs as estab- 
lished, if proofs are supposed to be unconditional - there is no such thing as general-acceptance conditional proof, because this is like saying "A is identical to B except it isn't" and "there are thongs identical that are more identical than other things identical". Who thinks that they are kidding whom here? Is this the "logic" of the "exact sciences" or of George Orwell's Animal farm? And, finally, should we be grateful to the technique of modus tollens logical inversions for uncovering the deadlock? And should we or should we not revert to it and use it more often and more conscientiously for checking up on our statements?

Anyone can see here that these equivalences are not unconditional, but need to be accompanied by a good nice reasonable commentary about the implication of fact, perception and temporal sequences, about necessity and sufficiency and conditionality, about aspects of theory, of subjectivity and objectivity, all about the observer and the observed.

Let us point out once again that logical inversions are supposed to be tautologies. The reader may immediately grasp that conjugate supposed tautologous pair of numbers, despite urgent need of further specifications regarding feature like the ones of which we already persistently made note and with all due caution regarding answering the stuff carrying the question marks:

- A1 and B4 are what instituted human perception and science hold as necessary fact;

- $\mathrm{A} 2$ and B2 are deranged garbage;

- $\mathrm{A} 3$ and B3 are superstitions, sorcery, and old wives' tales;

- A4 and B1 are just potential possibilities to maybe consider at will.

However, at no point should we lose view of the perspective that logical inversion, especially one carried out in modus tollens has strict mutatis mutandis rules. Combinations are not spontaneously unconditionally tautologous, in the sense that in one of the attempted pairing a slice of semantic charge has been put in or taken out or changed. But that is not all; people only too often make a capital error when they negligently just reverse the order, unduly turning necessities into potentialities and cases into generalities and all kinds of such confusions, thinking that they are making sense. This is where one must be very adamant in pointing out the impropriety of the conclusion. Contrary to some simple-folk-talk we all hear only too 
often, checking across the rest of the statements, the following "simplified" pseudo-inversion may NOT be considered as a tautology!

- "Every time there are maggots, there are eggs" does not necessarily imply that (and is not tautologous to):

- "Every time there are eggs, there are maggots".

As we shall demonstrate in what follows, alas, this is not a unique privilege of simple folk; major scientific fallacies exist right now exactly because (illogical) mis-inversions, such as the last one, are implicitly accepted as tautologies, whereas they are separate cases not necessarily having or sharing the same rules, the same models and the same truth-values as the original sentence.

\section{Scientific causalities?}

So many additional factors enter here in very treacherous manners regarding abstract theory and the scientifically orientated models. Let us choose one at random and watch it. What is cause and effect in, say, gravity? Things fall. That is an observable effect and a source of information, therefore an empirical cause of expressing a question and of formulating a problem. Why? Is there a cause in the spirit of a droit naturel like an analogue of the egg here? Alas no. Newtonian mechanics says the cause is gravity, and that gravity is a force and causes attraction / mutual acceleration. Is any of this anything like a natural cause like the egg? Absolutely not. Did something exist before? So? Should a bona fide epistemologist not demand of empiricism to empirically observe and record the natural cause like it does an egg? Where is the force? Is it perhaps in a ubiquitous decentralized neutral bearing 3D space of no focus, consisting of $0 \mathrm{D}$ points that are despite it $1 \mathrm{D}$ vectors that form a spatial field of directional points that are not directional, in this neutral space that has no focus but does have a centre of coordinates which is not a centre and is thus centralized but isn't?

And what is attraction and what is force? Is it an object? Definitely not! Is it a phenomenon? Definitely not! Is it observable? Definitely not! Was it before? Was it independent? Well, maybe not... Then how can it be an empirical cause like the egg? Is it even a cause? Not really; in a proper frame of mind of our being duly cool and distant about stuff, as epistemonic principle would wish us to be, what is not observable should not be a source of 
observation so it cannot even be a cause of information release. If we want to be cool, a page of equations and whole sets of tensors recording attraction and force is a mental model on paper of nothing more than systemic recording of an acceleration behaviour, a schematic systemic arrangement of mentally prior-posterior manifestations of abstract postulated patterns of relationships, totally unreal, nothing like the egg-type cause.

Where are we heading at? Watch! Why do things fall? Because there is gravity. Why is there gravity? Because things fall. And what is gravity? It is a force. And what is a force? The cause of acceleration. And why is there acceleration? Because there is force. And why is there force? Because we observe acceleration, don't you? OK then, what is the cause for the cause? It is a field. Why is there a field? Because I conclude there is acceleration. Why do I conclude that? Because things fall. OK, but WHY IS THERE gravity? Because it is a curvature in space-time. And how do you know? Here are the equations. And why are the equations what they are? Because space-time curves. And what is space-time? There you go again, I told you, here are the equations....

And then, suddenly no; it is space-time curvature, not a force, and that has been "proved by Einstein". If it has been proved, then it should be an abstract theorem and its proof must have been a tautology. But suppose the language here is loose and by proof we just mean verification. What was verified? Was it the cause, that was pre-extant, or was there a congruence of an abstract model established and (in a manner of speaking) "verified" as consistent to the observed behaviour? Why then isn't the said behaviour both (its own) cause and (its own) effect, or better said an auto-cause / auto-effect syndrome, if nothing in it existed before, outside and independently of what we observed? And if this physics is not Newtonian, and if the alleged space-time-curvature is an intrinsic feature of the universe's hyper-geometry, why does it cause acceleration? Or are some accelerations' causes not acceleration's causes? Or are some forces less forces than others? If yes, are we indeed taking walks on the wild side of George Orwell's Animal farm? If not, then what is the definition of force and non-force, since the Newtonian one we have been using is sometimes unusable? Where? Why? When? Where not? Why not? When not? Says who?

Conclusion: there is no equivalence of the natural empirical causality of our biological paradigm of seeds and mildew and eggs and hatchling chick- 
ens and maggots in spoiled meat in physical theory dealing in quasi-abstract or would-be-abstract models, because mathematical abstraction demands lack of semantic significance, whereas these physical formulations do mean things.

Are we, then, to conclude that if something is not directly observable -as in figures and strains of characters and symbols on "my" sheets of writing and "my" computer screens-, it stays hypothetical and cannot be construed as systemic cause and source, but only on the fringe, as a ghost hypothesis useful for description and systematization and study, but equivalent to the effect of some phantom mechanism, itself functional only as a similarly phantom cause of information? And what else would that mean about other "things" and "phenomena" not individually directly observable, such as say a single photon, but only "through their cumulative physical effects"? Or, worse, like dark matter which is an "unverified" side-effect of our equations, just to be tossed in to make them work? Would this, then, by this analysis, mean that such stuff would be "causes of information" towards "effects of description", totally upsetting the integrated normal image of natural cause and effect like egg and maggot? And would that lead to the apt conclusion that such things are not acceptable as empiricist science, let alone "exact", and cannot be construed either as necessary nor as sufficient, because there is no natural right applicable even on an honest-to-goodness mutatis mutandis basis? Are we saying this? Are we?

So, then, what is the cause? Is it the syndrome or is it the symptoms? And how much of the syndrome is aspects of the symptoms and vice versa? How much of it is in the before and how much is in the after? Is this total jumble, this loss of directionality of cause vs. effect, an inseparable mishmash of rationalist and magic thinking? Is it? And is an epistemologist entitled to dig up his/her stuff in this manner? Is he/she expected or required to do so? We are afraid that we shall have to leave these questions hanging at this point and proceed further, in the hope that some light will shine showing the way to a meaningful exit.

\section{Post Scriptum}

Have we managed to boggle the minds of the reader? If we have, it was neither intentional nor desired; our own minds have been boggled for the 
longest time as well. However, this tedious agonizing quasi-nihilistic trip has been delightful in a wildly perverse sort of way. How about expecting a sequel for it in the next issue, say... about... opening a new gate to... dimensions and geometry and whatever else that may bring?

\section{References}

Collins, H. M., \& Evans, R. (2002). The Third Wave of Science Studies : Studies of Expertise and Experience. Social Studies of Science 32/2 (April, 2(2), 235-296.

Latour, B., \& Woolgar, S. (1986). Laboratory Life: The Construction of Scientific Facts. Princeton University Press. Retrieved from https://books.google.gr/books?id=vJ-JueUwptEC\&pgis=1\&redir esc=y

Lekkas, D. (2003). Towards an epistemonic theoretical surveillance of dance. In Surveillance of Greek Music \& Dance (pp. 204-206). Greek Open University.

Papageorgiou, K. G., \& Lekkas, D. (2018). On the Methodology of the Analytic Method: Historical Account, Epistemological Suggestions, Stages. Epistēmēs Metron Logos, (1).

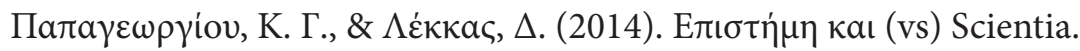

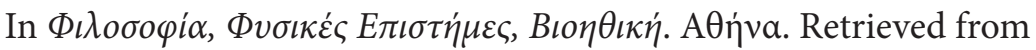
http://deeaef.gr/wp-content/uploads/2016/04/Papageorgiou-Lekkas-full-text. pdf 